\title{
Comparison of Different Approaches to Manufacturing Process Optimization
}

\author{
Pavel Mach, Seba Barto \\ Department of Electrotechnology \\ Czech Technical University in Prague, Faculty of Electrical Engineering \\ Prague, Czech Republic \\ mach@fel.cvut.cz
}

\begin{abstract}
There are many process variables, which more or less influence quality of a manufacturing process, and there are many parameters of a product, which constitute its quality. The control of variables of a manufacturing process for achievement of requested quality of a final product is a multi-parametric task. It is paid great effort to find such the way, which might lead to the most rapid solution. The use of full factorial experiments, fractional factorial experiments and Taguchi approach were compared from the point of view of number of experiments requested. It was found that the Taguchi orthogonal arrays are the most efficient method for higher number of process factors and that the full factorial experiments request the highest number of experiments from all methods under test.
\end{abstract}

Keywords-full factorial experiments; fractional factorial experiments; Taguchi orthogonal arrays

\section{INTRODUCTION}

Contemporary production organization must have the ability to produce a variety of high-quality products with low prices to be successful on the market. There are many ways how to optimize production quality. It must be stressed that quality improvement is most effective when it is an integral part of the product and process development cycle.

Experimental design is the earliest stage of the development cycle, where new products are designed, existing products are improved, and manufacturing process is optimized. Proper application of an experimental design methodology leads to design of products, which are easier to manufacture, have enhanced field of performance and have higher quality and reliability.

Manufacturing processes in electronics are controlled with several factors usually. These variables have different influence on the final result of a process. Some of them have high influence, some of them weak only. The process can also be influenced with interactions between these factors.

Experimental design methods are used either in process development or process troubleshooting for improvement process performance, or to obtain process, which is robust and insensitive to external sources of variability [1]. Using other term, because quality is inversely proportional to variability, experimental design methods make possible to develop a process resistant against influences, which would worsen its quality.
Experimental design is an active statistical method. We perform series of experiments on the process making changes in its inputs and analyze corresponding changes in the outputs. Results of analysis of these mutual relationships lead to improvement of process quality. Therefore experimental design is a critically important engineering tool for improvement of manufacturing process quality.

Significant role has this methodology also in the field of development of new processes. Application of experimental design in early stage of process development can result in improvement of yield, in reduction of variability of final product parameters, in shorter time of production and in reduction of total costs.

Experimental design is also used in evaluation and mutual comparison of different solutions of structures of processes in the stage of new processes development, in evaluation of different materials alternatives, in analyzes leading to determination of key process parameters, which influence the output parameters of a product dominantly and in determination of process parameters, which influence its performance dominantly.

Design of factorial experiments can be carried out in two versions: as full factorial experiments and as fractional factorial experiment. These modifications differ in number of experiments and in depth of analysis. Fractional factorial experiments need lower volume of experimental work, but influence of some interactions of factors is omitted.

Since introduction of design of experiments there were many developments with this technique, but its practical use was low. Taguchi attempted to improve this situation. He showed that design of experiments can be used not for quality improvement only, but that it is possible to use it for quantification of improvement evaluated according to the volume of saved money. He standardized application method as well and made this technique easier for the use this way. $\mathrm{He}$ developed number of orthogonal arrays, each of which is directed for the use in a number of experimental situations. Together with presentation of these arrays he presented new way for results analysis. Typical for this analysis is that it offers approach, which is robust to influence of uncontrollable factors.

Effectiveness of different methods for improvement of manufacturing process quality must be evaluated, instead other 
parameters, according to the number of experiments, which are requested by these methods. This angle is very important, because many experiments are joined with material losses, many of them are expensive and all experiments are time consuming. Therefore it is necessary to weigh different methods for process optimization from two aspects: which will be the total costs joined with application of a method, which was chosen and which quality will have the result if this method is applied. Optimization of these two aspects is not easy.

\section{THEORETICAL BACKGROUND}

\section{A. Full Factorial Experiments}

General model of a manufacturing process has many input factors and some output factors (see Fig. 1). Input factors are controllable and uncontrollable. Input factors can also been quantitative or qualitative. Quantitative factors can be measured and the result can be expressed as a number. Typical quantitative factors are electric voltage, electric current, weight, high, pressure, humidity and many others. Qualitative factors either can be measured, but it is impossible to express them as numbers, or they cannot be measured. Typical qualitative factors are shape, color, smell and others. Design of experiment is mostly concerned with quantitative factors, but qualitative factors are also used for process optimization sometimes.

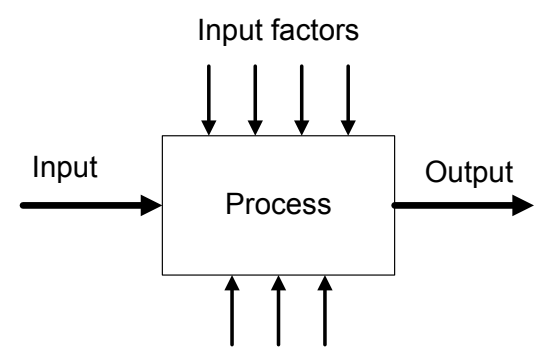

Input factors

Figure 1. General model of a manufacturing process

Optimization of a process based on design of experiments consists of following steps:

- Recognition and definition of a problem. This part is very important and is not easy in many cases. Clear definition of a problem contributes to better process understanding and supports solution of a problem.

- Choice of factors and determination of ranges of their changes, their means and their acceptable variability.

- Selection of response variable. This step is easier in comparison with the step no. 1 . Variable must provide useful information about the process. Response variable can be defined with respect to the important parameters of a final product, with respect to the ideas of customers, with respect to the yield of a process or from other angles.

- Decision about experimental design. This step involves decision about sample size, about number of runs, and climatic and other ambient conditions in which experiments are performed.

- Performing the experiment.

- Data analysis. If the experiments were correctly designed and if they were correctly performed, then types of statistical methods for data processing are good known. There is wide offer of software tools for statistical data processing as well.

- Conclusions. Results of the data analysis must draw practical conclusions about the results and recommend actions necessary for improvement.

Factorial experiments are based on examination of factor effects. These effects are defined as change of responses caused by changes of the levels of factors. Such the effects are called main effects. However, for example, if a two-factorial experiment will be examined, effect of the factor A will depend on level of the factor $\mathrm{B}$. Therefore interaction $\mathrm{AB}$ must be examined as well.

Schematic diagram for two-factorial experiment is shown in Tab. 1

TABLE I. Design of factorial experiments for two factors

\begin{tabular}{|c|c|c|c|c|c|}
\hline & & \multicolumn{4}{|c|}{ Factor B } \\
\hline & $\begin{array}{c}\text { Level of } \\
\text { factor }\end{array}$ & 1 & 2 & $\ldots$ & b \\
\hline \multirow{3}{*}{ 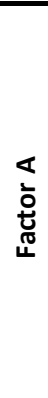 } & 1 & $\begin{array}{l}\text { y111, } \\
\text { y112,..., } \\
\text { y11n }\end{array}$ & $\begin{array}{l}y 121, \\
y 122, \ldots, \\
y 12 n\end{array}$ &. & $\begin{array}{l}y 1 b 1, \\
y 1 b 2, \ldots, \\
y 1 b n\end{array}$ \\
\hline & 2 & $\begin{array}{l}\text { y211, } \\
\text { y212, ... } \\
\text { y21n }\end{array}$ & $\begin{array}{l}y 221, \\
y 222, \ldots \\
y 22 n\end{array}$ & $\ldots$ & $\begin{array}{l}\mathrm{y} 2 \mathrm{~b} 1, \\
\mathrm{y} 2 \mathrm{~b} 2, \ldots \\
\mathrm{y} 2 \mathrm{bn}\end{array}$ \\
\hline & a & $\begin{array}{l}\text { ya11, } \\
\text { ya12,... } \\
\text { ya1n }\end{array}$ & $\begin{array}{l}\text { ya21, } \\
\text { ya22, ... } \\
\text { ya2n }\end{array}$ & $\ldots$ & $\begin{array}{l}\text { yab1, } \\
\text { yab2, ... } \\
\text { yabn }\end{array}$ \\
\hline
\end{tabular}

Where $1 \ldots$ a - levels of factor A, $1 \ldots$ b- levels of factor $\mathrm{B}, \mathrm{n}$ - number of replications of experiments.

Response $y$ has three indexes in every cell. The first one is the level of the factor $A$, the second one is the level of the factor $B$ and the third one is number of experiment replications.

In collecting the data, a.b.n observations would be run in random order. It means that two-factorial experiments are fully randomized.

Models found with the use of two-factorial experiments are linear models usually. Such the model can be described using following equation: 
2010 IEEE 16th International Symposium for Design and Technology in Electronic Packaging (SIITME)

$$
y_{i j k}=\mu+\alpha_{i}+\beta_{j}+(\alpha \beta)_{i j}+\varepsilon_{i j k}
$$

Where $\mu \ldots$ overall mean, $\alpha_{i} \ldots$ i-th effect of factor $\mathrm{A}, \beta_{j} \ldots$ $\mathrm{j}$-th effect of factor $B,(\alpha \beta)_{i j} \ldots$ effect of interaction between $A$ and $B$ and $\varepsilon_{i j k} \ldots$ a random error. Zero hypothesis testing effect of factors $A$ and $\mathrm{B}$ and interaction $A B$ is tested usually.

Mathematical processing of designed experiments is carried out using analysis of variance. First total sum of squares of deviations of measured values from the oveall mean is calculated:

$$
S_{T O T}=\sum_{i=1}^{a} \sum_{j=1}^{b} \sum_{k=1}^{n}\left(y_{i j k}-\overline{y_{T O T}}\right)^{2}
$$

Where $y_{i j k} \ldots$ measured value in $\mathrm{i}$-th raw and $\mathrm{j}$-th column on $\mathrm{k}$-th replication of an experiment, $\overline{y_{T O T}}=\mu \ldots$ oveall mean. The overall mean is calculated as follows:

$$
\overline{y_{\text {TOT }}}=\mu=\frac{\sum_{i=1}^{a} \sum_{j=1}^{b} \sum_{k=1}^{n} y_{i j k}}{a b n}
$$

Then sum of squares of deviations caused by the change of the factor $A$ from level 1to level $a$ is calculated at first, then sum of squares of deviations caused by the change of the factor $B$ from level 1 to level $b$ is calculated and then sum of squares caused by interaction $A B$ when level of the factor $A$ is changed from 1 to $a$ and level of the factor B from 1 to $b$ is calculated.

As the last step the residuum sum of squares $S_{E}$ is found. This value describes reproducibility of a process.

It follows from the schematic diagram of designed experiments (see Tab. 1) that overall $n$ values are measured in every cell. For example, for the cell in raw for level 1 of the factor $A$ and in column for level 1 of the factor $B$ responses $y_{111}$ to $y_{11 n}$ are measured, because the experiment is n-times replicated. For calculation of residuum sum of squares first mean of responses $\overline{y_{i j}}$ for the cell $i j$ is found. Then following squares of differences are calculated:

$$
D_{i j}=\sum_{k=1}^{n}\left(y_{i j k}-\overline{y_{i j}}\right)^{2}
$$

Where

$$
\overline{y_{i j}}=\frac{\sum_{k=1}^{n} y_{i j k}}{k}
$$

Then residuum sum of squares is calculates according to the equation:

$$
S_{E}=\sum_{i=1}^{a} \sum_{j=1}^{b} D_{i j}
$$

Total number of experiments, which must be accomplished, is given by the equation:

$$
N E=a \cdot b \cdot n
$$

Number of experiments is high for higher number of factors and levels of factors.

\section{B. Fractional Factorial Experiments (FEE)}

When the number of factors increases, the number of runs required increases rapidly. For example, $2^{6}$ experiments require 64 runs. If every run is 10 times repeated, the total number of experiments, according to the equation (7), is 640. In this design 6 degrees of freedom represent main effects and other degrees represent more-factors interactions.

If designed experiment of the k-type (let us assume, for example, experiment of the type $2^{k}$ ) will be analyzed and if it will be assumed that main effects are dominant and that some higher-order interactions can be neglected from the point of view of a process description, then fractional factorial experiments involving fewer than complete set of $2^{\mathrm{k}}$ runs can be used to obtain information about the main effects and some lower-order interactions.

There are many different types of fractional factorial experiments. The one-half fraction of the $2^{k}$ design is used very often.

A one-half fraction of the $2^{k}$ design contains $2^{k-1}$ runs. Let us assume that $k=3$. Design with the runs, which yield plus on the $A B C$ effect, are used usually. Therefore $A B C$ is called generator of this particular fraction.

When the table of plus minus signs for factorial design of the type $2^{3}$ is analyzed, it is possible to find following estimates of main effects:

$$
\begin{aligned}
& A=0,5(a-b-c-a b c) \\
& B=0,5(-a+b-c+a b c) \\
& C=0,5(-a-b+c+a b c)
\end{aligned}
$$

However, it is also possible to find that

$$
\begin{aligned}
& B C=0,5(a-b-c-a b c) \\
& A C=0,5(-a+b-c+a b c) \\
& A B=0,5(-a-b+c+a b c)
\end{aligned}
$$

If equations (8) and (11); (9) and (12); and (10) and (13) will be compared, it will be found that A estimates $\mathrm{BC}, \mathrm{B}$ estimates $\mathrm{AC}$ and $\mathrm{C}$ estimates $\mathrm{AB}$. Effects, which have this property, are called aliases.

It is possible to decrease the number of runs with the use of these aliases. Finally, for this one-half fraction of the $2^{k}$ design, is the result in decrease of the number of runs requested given by the formula $2^{k-1}$. For example, if the $k=3$, the number of requested runs for full factorial experiments of the type $2^{k}$ is 8 , whereas for this one-half design four runs only are requested. It is obviously that when $k$ will increase, effectiveness of fractional factorial experiments will increase in comparison with the full factorial experiments. 


\section{Taguchi Orthogonal Arrays}

Methodology of Taguchi is next way for process quality improvement. Taguchi calculated tables of orthogonal arrays [2]. These arrays are designed for different types of experiments. Some of them for factors having two levels and for low number of factors are presented in Tab. 2

TABLE II. Taguchi Orthogonal Arrays for two-level factors

\begin{tabular}{|c|c|c|c|c|}
\hline $\begin{array}{c}\text { Type of } \\
\text { array }\end{array}$ & L4 & L8 & L9 & L12 \\
\hline $\begin{array}{c}\text { Number } \\
\text { of } \\
\text { factors }\end{array}$ & 2 or 3 & 4 to 7 & 4 to7 & 8 to 11 \\
\hline
\end{tabular}

Three types of factors are used for Taguchi analysis: controlled factors, factors, for which the process must be terminated, when it is necessary to change it and a noise factor. Analysis is carried out using analysis of variance [3].

\section{PRACTICAL USE OF DESCRIBED METHODS}

An example for practical use of Taguchi orthogonal array of the type L9 is presented. The Taguchi approach was applied for improvement of a process of wave lead-free soldering. Number of pads without bridges is taken as response.

Controlled factors of this process are:

- Time of a contact of melted solder with the pad and lead of a component (factor A).

- Pre-heating temperature (factor B).

- Volume of flux (factor C).

Factor, for which the process must be terminated, when it is necessary to change it, is the solder temperature expressed as the time needed to the increase/decrease the temperature (factor D). It is necessary to add noise factor as well in the analysis.

Taguchi orthogonal array of the type L-9 is shown in Tab. III. Every run is one times repeated.

TABLE III. Taguchi Orthogonal Arrays

\begin{tabular}{|c|c|c|c|c|c|c|}
\hline \multicolumn{5}{|c|}{ Control factors } & \multicolumn{2}{c|}{ Pads without bridges } \\
\hline Group & A & B & C & D & Exp. \#1 & Exp. \#2 \\
\hline 1 & 1 & 1 & 1 & 1 & 198 & 196 \\
\hline 2 & 1 & 2 & 2 & 2 & 188 & 198 \\
\hline 3 & 1 & 3 & 3 & 3 & 171 & 161 \\
\hline 4 & 2 & 1 & 2 & 3 & 200 & 199 \\
\hline 5 & 2 & 2 & 3 & 1 & 169 & 192 \\
\hline 6 & 2 & 3 & 1 & 2 & 192 & 196 \\
\hline 7 & 3 & 1 & 3 & 2 & 196 & 192 \\
\hline 8 & 3 & 2 & 1 & 3 & 200 & 199 \\
\hline 9 & 3 & 3 & 2 & 1 & 178 & 172 \\
\hline
\end{tabular}

Dispersion analysis showed that the most significant parameter for bridging is the time of contact between pad and solder $(38 \%)$, lower influence has pre-heating temperature (28\%), volume of flux (24\%) and solder temperature $(10 \%)$ Total number of experiments was 18 . In the case of the use of the full factorial experiments the total number of experiments is 32 and for fractional factorial experiments of one-half type this number is 16 .

The number of experiments requested for FEE and Taguchi approach is examined for the number of control factors between 2 and 13 and for variables having 2 levels and 3 levels. The result is shown in Fig. 2.

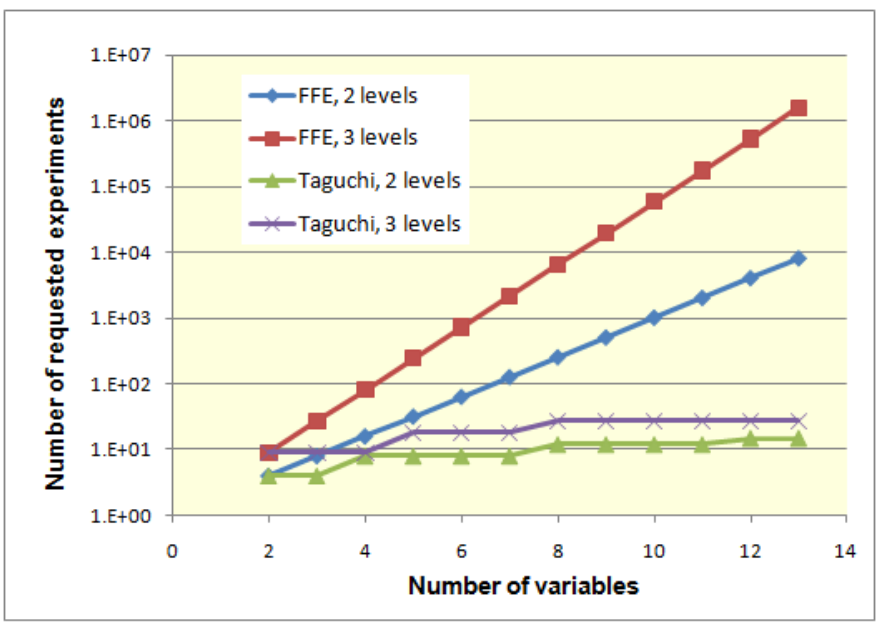

Figure 2. Comparison of number of experiments needed for full factorial experiments and Taguchi approach. Number of experiments for fractional factorial experiments depends on the type of these experiments.

\section{CONCLUSIONS}

The use of full factorial experiments, fractional factorial experiments and Taguchi approach for analysis of manufacturing processes is shown. Efficiency of these methods is evaluated from the point of view of the number of experiments, which are requested by different approaches.

It is shown that the higher is the number of process variables and levels of these variables, the higher is efficiency of the Taguchi approach in comparison with full factorial experiments and fractional factorial experiments. It is shown how inevitably is the use of some of these methods for improvement of process quality.

\section{REFERENCES}

[1] D. C. Montgomery, "Introduction to statistical quality control”. J. Wiley and Sons. 2001

[2] G. Taguchi, S. Chowdhury, Y. Wu: „Taguchi's Quality Engineering Handbook“. J. Wiley and Sons. 2005.

[3] R. K. Roy: „Design of Experiments Using The Taguchi Approach: 16 Steps to Product and Process Improvement“. J. Wiley and Sons. 2001. 